\title{
Acción del Fenoterol sobre la Contractilidad del Utero Humano Grávido
}

Cifuentes, Rodrigo*

\section{Introducción}

Existe acuerdo, en general, en que los agentes beta-estimulantes poseen la propiedad de inhibir la contractilidad uterina en la mujer grávida, lo cual tiene amplia aplicación en el manejo de algunas entidades clínicas como la amenaza de parto prematuro (1) o el sufrimiento fetal agudo intraparto (2).

Igualmente, ha sido demostrado que la presencia de algunos radicales en estas aminas simpaticomiméticas incrementan la afinidad de ellas sobre los receptores beta, especialmente los llamados beta-2. Entre los radicales que producirán un aumento en los efectos beta-estimulantes se puede mencionar el anillo fenólico, con el hidróxido en posición para, el cual constituye una de las estructuras básicas del fenoterol.

El objetivo del presente trabajo fue el de estudiar el efecto del fenoterol sobre la contractilidad uterina y el aparato cardiovascular materno-fetal.

\section{Sujetos y Métodos}

Se estudió la acción del fenoterol (Partusistén R) en 20 mujeres con embarazo de 26 a 41 semanas de amenorrea. De este grupo, en 1.0 pacientes se realizó registro continuo y simultáneo de contractilidad uterina y frecuencia
Mora, Jaime Dario*

cardíaca fetal (F.C.F.). Después de media hora de buen registro de la contractilidad uterina se administró el fármaco mencionado a la dosis de $10 \mathrm{mgr}$. vía sublingal, continuando el registro por lo menos una hora más.

En el grupo de pacientes en quienes no se monitorizó la contractilidad uterina ni la FCF, estos parámetros fueron controlados por los métodos clínicos convencionales.

Análisis de los registros. Para cada caso registrado se seleccionaron 2 períodos de 30 minutos cada uno inmediatamente antes $\mathrm{e}$ inmediatamente después de la administración de la droga.

En cada período se analizó la contractilidad uterina en sus componentes de interisidad (en $\mathrm{mmHg}$ ), frecuencia (en 10 minutos), tono (en $\mathrm{mmHg}$ ) y actividad uterina (en Unidades Montevideo). Se cuantificó el promedio de FCF antes y después de la administración de la droga, así como la FC y la presión arterial maternas.

En cada registro se calculó el promedio para cada una de las variables mencionadas, correspondientes a los dos períodos considerados. La significación de las diferencias entre ambos períodos fue analizada con el método de " $t$ " para muestras dependientes.

\footnotetext{
* Unidad de Fisiología de la Reproducción. Departamento de Obstetricia y Ginecología. Universidad del Valle, Cali, Colombia.
} 


\section{Resultados}

Durante la administración del fenoterol se objetivó una disminución en todos los parámetros de la contractilidad uterina respecto de los valores previos (fig. 1). Esta disminución fue estadísticamente significativa (p. 0.05) para la intensidad, frecuencia y actividad uterinas, no así para el tono (Tabla I), figs. 1 y 2 .

Las frecuencias cardiacas fetal y materna se incrementaron significativamente después de la administración del fármaco (fig. 3). Sin embargo, el aumento fue más marcado en la frecuencia cardiaca materna (22 latimin) que en la FCF (7 lat/min).

La presión arterial sistólica no tuvo modificaciones, mientras que la diastólica tuvo un ligero descenso que no fue significativo.

\section{Discusión y Comentarios}

La efectividad del fenoterol como inhibidor de las contracciones uterinas lograda en este trabajo, concuerda con resultados publicados anteriormente por diversos investigadores tanto en humanos $(3,4)$ como en animales de experimentación (5).

El mecanismo a través del cual actúan los fármacos beta miméticos no está bien claramente establecido. Sin embargo, se conoce que la estimulación de los beta-receptores incrementa el contenido intracelular del AMP cíclico en el músculo uterino por un efecto estimulante de la adenil ciclosa (6). El aumento del CAMP está bién correlacionado además, con un efecto relajante muscular uterino (7). Por otra parte la beta-estimulación desencadena un aumento en la adenil ciclosa no solamente en la fibra muscular uterina sino también en todos los tejidos que tengan receptores beta, por lo cual se producen fundamentalmente efectos cardioaceleradores, vasodilatadores e hipotensivos. Estos disturbios cardiovasculares han limitado considerablemente el uso de estos fármacos.

Los resultados presentados con fenoterol demuestran que es un potente inhibidor de la contractilidad uterina con efectos relativamente menores sobre el aparato cardiovascular. Es así, como a la dosis de $10 \mathrm{mgr}$. por vía sublingual se obtuvo una significativa disminución en la actividad uterina, poco incremento en la frecuencia cardíaca materno-fetal, ningún cambio significativo en la presión arterial materna y prácticamente ningún síntoma subjetivo. Debido a lo anterior podría sugerirse el uso del fenoterol en aquellos casos en los cuales esté indicada la inhibición de la contractilidad uterina, como las mencionadas al comienzo del presente trabajo.

\section{T A B L A I}

\section{Efecto del fenoterol sobre la contractilidad uterina y la frecuencia cardiaca materno-fetal}

\begin{tabular}{lrrrr}
\hline & \multicolumn{1}{c}{ Antes* } & Después $*$ & "t" & $\mathrm{P}$ \\
\hline Actividad uterina & $220.5 \pm 32.9$ & $73.3 \pm 21.2$ & 3.4 & 0.01 \\
Frecuencia & $5.3 \pm 0.9$ & $3.3 \pm 0.7$ & 3.2 & 0.02 \\
Intensidad & $39.7 \pm 5.3$ & $19.8 \pm 5.1$ & 4.9 & 0.001 \\
Tono & $8.1 \pm 2.8$ & $3.3 \pm 1.6$ & 1.5 & N.S. \\
F. C. F. & $151.6 \pm 2.2$ & $157.8 \pm 2.9$ & 2.7 & 0.05 \\
F. C. M. & $79.0 \pm 3.3$ & $101.3 \pm 5.7$ & 2.8 & 0.05 \\
\hline
\end{tabular}

\pm E.S. 


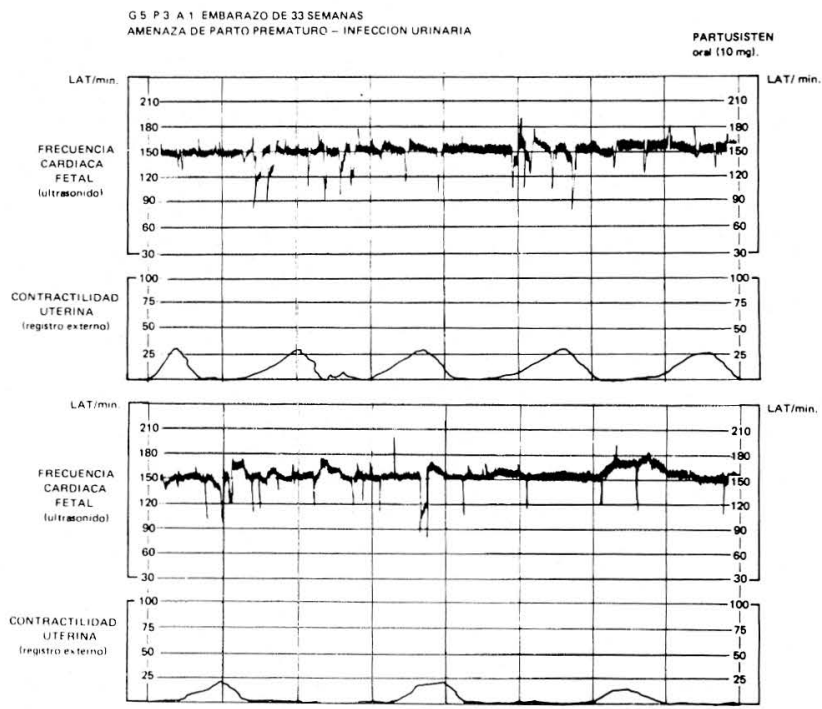

FIGURA N 1 - Registro de contractilidad uterina y frecuencia cardiaca fetal en una embarazada con amenaza de parto prematuro. Nótese en la parte superior la contractilidad uterina espontánea y en la inferior, el comienzo de la útero inhibición inmediatamente después de administrar fenoterol

(Partusistén) sublingual.

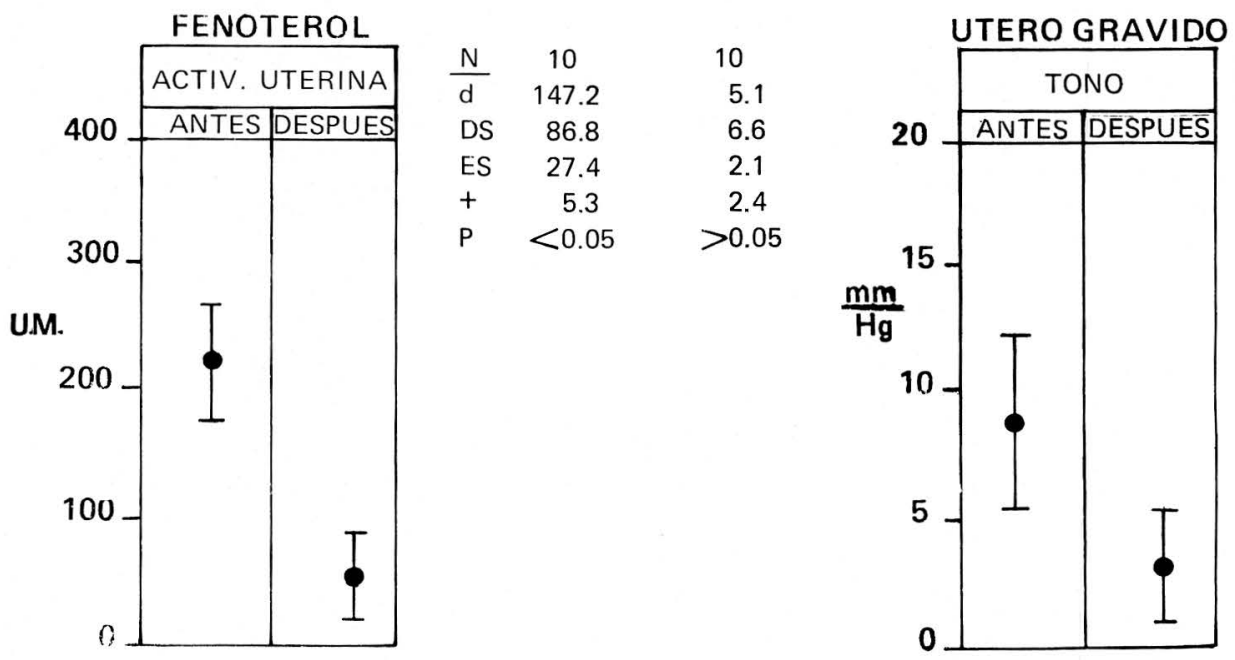

FIGURA N: 2 - Actividad (frecuencia por intensidad) y Tono (en $\mathrm{mmHg}$ ) ątes y después de la administración sublingual de fenoterol. La disminución en el último parámetro mencionado no fue significativa. Sin embargo la intensidad, la frecuencia y actividad uterina disminuyeron considerablemente. 


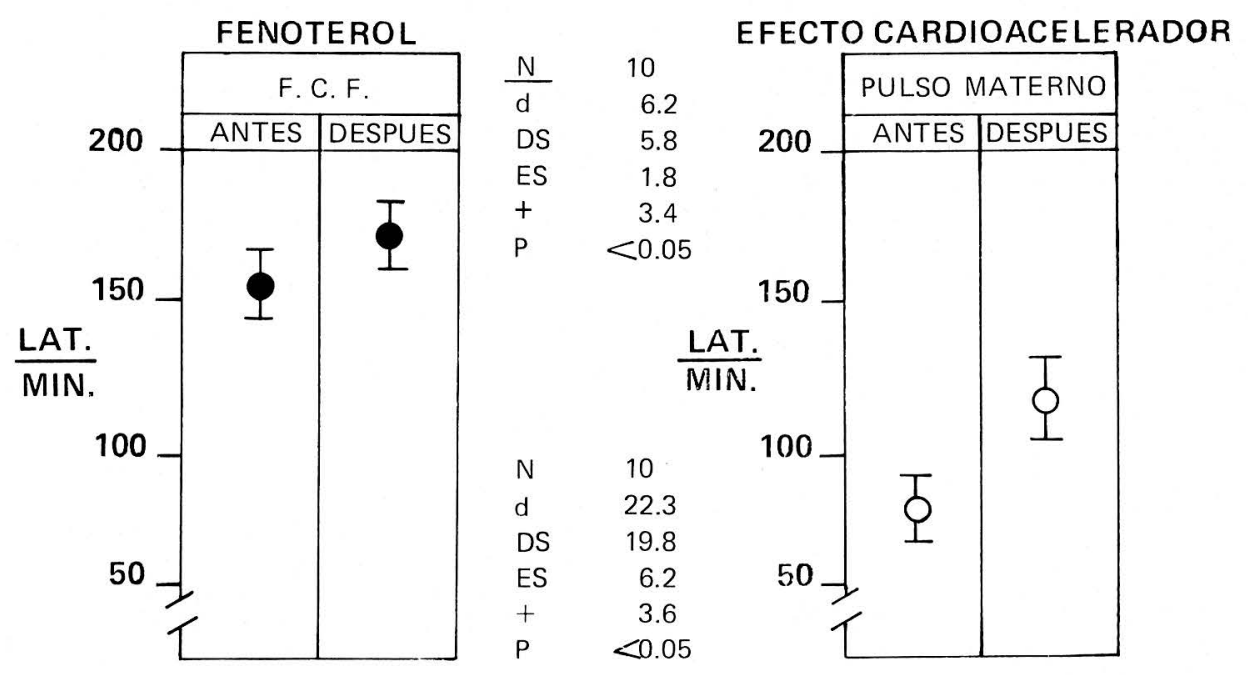

FIGURA N: 3 - Efecto acelerador del fenoterol sobre la frecuencia cardiaca fetal y materna Aunque se presentó un incremento significativo, el aumento promedio en la F. C. F. fue solamente de 6.2 lat/min. La F. C. materna aumentó 22.3 lat/min. en prornedio.

\section{Resumen}

Se estudió el efecto de un fármaco estimulante selectivo de los receptores beta-2 sobre la contractilidad uterina en un grupo de 20 mujeres con embarazo entre 26 y 41 semanas de amenorrea.

Todas las pacientes recibieron fenoterol por vía sublingual a las dosis única de $10 \mathrm{mgr}$. En la mitad de los casos se realizó registro continuo de contractilidad uterina y frecuencia cardíaca fetal. La presión arterial y frecuencia cardíaca maternas se controlaron por métodos convencionales. Se analizaron cuantitativamente los registros antes y después de la administración del fármaco. En la mayoría de los casos se encontró una disminución estadísticamente significativa de los parámetros de contractilidad uterina analizados, así como ligera elevación en la frecuencia cardiaca materna y fetal.

Finalmente, se sugiere el empleo del fenoterol en aquellas entidades en que esté indicado hacer inhibición de la contractilidad uterina, ya que por su acción selectiva sobre los adrenoceptores beta-2, se obtiene un buen efecto úteroinhibidor con mínimos efectos cardiovasculares.

\section{PHENOTEROL ACTION OVER HUMAN GRAVID UTERUS CONTRACTILITY}

\section{Summary}

The effects of a selective stimulant drug of Beta-2 receptors over the uterine contractility were analyzed in a group of 20 (twenty) pregnant women between 26 and 41 weeks of amenorrhea.

All the patients were given phenoterol by sublingual way at the unique do- 
se of $10 \mathrm{mgr}$. In half of the cases uterine contractility and fetal cardiac fre. quency were continuously recorded. Maternal blood-pressure and cardiac frequency were controlled through traditional methods. The records were quantitatively analyzed both before and after the drug was administered. In most cases a statistically significant reduction of the analyzed uterine contractility guidelines was observed, as

\section{Bibliografía}

1 SCHWARCZ. R. (h), PUEYRREDON, H. ALTHABE, O. Tratamiento de la amenaza de parto prematuro con clorhidrato de etiladrianol (Effortil). Rev. Arg. Ginec. Obstet. 1: 148, 1970.

2 CALDEYRO-BARCIA, R., MAGAÑA, J. M., CASTILLO, J. B. Nuevo enfoque para el tratamiento del sufrimiento fetal agudo intraparto. Arch. Ginec. Obstet. 24: 15, 1965.

3 MOSLER, K. H., LINKA, F., DORNHOFER, W. Tocolytic therapy in Obstetrics. J. Perinat. Med. 2: 3, 1974. well as a light increase in maternal and fetal cardiac frequencies.

Finally, it is recommended to use phenoterol in those entities where inhibition of uterine contractility is indicated, since due to its selective action over Beta-2 adrenoreceptors, a good uterus inhibitor effect is attained with minimum cardiovascular effects .

4 LIPSHITZ, J., BAILLIE, P. The effects of the fenoterol hydrobromide aerosol on ute rine activity and the cardiovascular system. Brit. J. Obstet. Gynaec. 83: 864, 1976.

5 LAUERSEN, N., WILSON, K., FUCHS, F. The effect of berotec (th-1165-a) on spontaneous and induced uterine activity in the pregnant baboon. Am. J. Obstet. Gynec. 121: $597,1975$.

6 ROBINSON, G. A., BUTCHER, R. W., SUTHERLAND, E. W.: Cyclic AMP, Annu. Rev. Biochem. 37: 149, 1968.

7 MARSHALL, J. M. Effects of catecholamines on the smooth muscle fibers in the rat. Ann. Rev. Pharmacol. 13: 19, 1973. 